\title{
Novel type of linear mitochondrial genomes with dual flip-flop inversion system in apicomplexan parasites, Babesia microti and Babesia rodhaini
}

\author{
Kenji Hikosaka ${ }^{1,2}$, Naotoshi Tsuji ${ }^{3}$, Yoh-ichi Watanabe ${ }^{2}$, Hiroe Kishine ${ }^{4}$, Toshihiro Horii ${ }^{5}$, Ikuo Igarashi',
} Kiyoshi Kita ${ }^{2^{*}}$ and Kazuyuki Tanabe ${ }^{1,5^{*}}$

\begin{abstract}
Background: Mitochondrial (mt) genomes vary considerably in size, structure and gene content. The mt genomes of the phylum Apicomplexa, which includes important human pathogens such as the malaria parasite Plasmodium, also show marked diversity of structure. Plasmodium has a concatenated linear mt genome of the smallest size (6-kb); Babesia and Theileria have a linear monomeric mt genome (6.5-kb to 8.2-kb) with terminal inverted repeats; Eimeria, which is distantly related to Plasmodium and Babesia/Theileria, possesses a mt genome (6.2-kb) with a concatemeric form similar to that of Plasmodium; Cryptosporidium, the earliest branching lineage within the phylum Apicomplexa, has no mt genome. We are interested in the evolutionary origin of linear mt genomes of Babesial Theileria, and have investigated $\mathrm{mt}$ genome structures in members of archaeopiroplasmid, a lineage branched off earlier from Babesia/Theileria.
\end{abstract}

Results: The complete mt genomes of archaeopiroplasmid parasites, Babesia microti and Babesia rodhaini, were sequenced. The mt genomes of B. microti $(11.1-\mathrm{kb})$ and B. rodhaini $(6.9-\mathrm{kb})$ possess two pairs of unique inverted repeats, IR-A and IR-B. Flip-flop inversions between two IR-As and between two IR-Bs appear to generate four distinct genome structures that are present at an equi-molar ratio. An individual parasite contained multiple $\mathrm{mt}$ genome structures, with 20 copies and $2-3$ copies per haploid nuclear genome in B. microti and B. rodhaini, respectively.

Conclusion: We found a novel linear monomeric mt genome structure of B. microti and B. rhodhaini equipped with dual flip-flop inversion system, by which four distinct genome structures are readily generated. To our knowledge, this study is the first to report the presence of two pairs of distinct IR sequences within a monomeric linear mt genome. The present finding provides insight into further understanding of evolution of mt genome structure.

Keywords: Mitochondrial genome, Babesia/Theileria, Piroplasma, Apicomplexa, Flip-flop inversion

\section{Background}

Mitochondria, organelles essential for energy transduction, are present in almost all eukaryotes and have their own genome. Like nuclear genomes, mitochondrial (mt) genomes vary considerably in size, structure, and gene content [1]. There are two major mt genome forms: circular and linear. Circular forms are present in animal $\mathrm{mt}$ genomes with sizes ranging from $15 \mathrm{~kb}$ to $20 \mathrm{~kb}$ and

\footnotetext{
* Correspondence: kitak@m.u-tokyo.ac.jp; kztanabe@biken.osaka-u.ac.jp ${ }^{2}$ Department of Biomedical Chemistry, Graduate School of Medicine, The University of Tokyo, 7-3-1 Hongo, Bunkyo-ku, Tokyo 113-0033, Japan Full list of author information is available at the end of the article
}

gene arrangements in the genomes are remarkably stable [2]. Some animal circular mt genomes are composed of more than two chromosomes or minicircles (e.g., the sucking louse Pediculus humanus, [3]). Circular forms are also found in higher-plant mt genomes. A higher-plant $\mathrm{mt}$ genome is characterized by a multipartite structure, which contains several subgenomic circular molecules with various organizational features, with sizes ranging from $200 \mathrm{~kb}$ to $2400 \mathrm{~kb}$ [4].

Linear $\mathrm{mt}$ genomes are found in diverse, unrelated organisms and have terminal inverted repeat (TIR) on both ends [5]. In some organisms, the mt genomes are

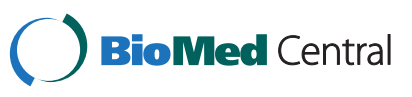


divided into multiple chromosomes (e.g., the colorless green alga Polytomella parva, [6]), or several hundred chromosomes (e.g., the ichthyosporean Amoebidium parasiticum, [7]). In the phylum Apicomplexa, which includes important pathogens such as the causative agents of malaria (Plasmodium), coccidiosis (Eimeria), and piroplasmosis (Babesia and Theileria), the $\mathrm{mt}$ genome structure is also diverse. Monomeric linear $\mathrm{mt}$ genomes with TIR on both ends are found in the Babesia and Theileria genera [8]. The Babesia/Theileria mt genomes are from $6.6 \mathrm{~kb}$ to $8.2 \mathrm{~kb}$ in size and encode only three protein coding genes (cytochrome $c$ oxidase subunit I [coxl] and III [cox3] and cytochrome $b[c o b]$ ) in addition to 24 fragmented small subunit (SSU) and large subunit (LSU) ribosomal RNA (rRNA) sequences $[8,9]$. Plasmodium, closely related to Babesia/Theileria [10], has the minuscule 6-kb tandemly repeated linear or concatenated $\mathrm{mt}$ genome, which encodes the same three protein coding genes as Babesia/Theileria [11,12]. The gene arrangements and transcriptional direction are however different from Babesia/Theileria. Furthermore, SSU and LSU rRNA genes of Plasmodium are highly fragmented with 27 pieces $[9,13]$ and the pattern of fragmentation differs from Babesia/Theileria [8,9]. Eimeria, which is distantly related to Babesia/Theileria and Plasmodium, possess a concatemeric form and contains the same three protein-coding genes and 20 rRNA gene fragments as Plasmodium $[9,14,15]$. A recent phylogenetic study suggests that a concatenated form appears to be the ancestral $\mathrm{mt}$ genome structure in the phylum Apicomplexa, with the monomeric linear form of Babesia/Theileria having evolved in the lineage [14]. We are interested in the evolution of linear mt genomes of Babesia/Theileria, and investigated mt genome structure of the rodent piroplasms, Babesia microti and Babesia rodhaini, which belong to archaeopiroplasmids group, a lineage which branched off earlier from Babesia/Theileria [16]. Results revealed that both B. microti and B. rodhaini have a monomeric linear $\mathrm{mt}$ genome, in which two pairs of unique IR sequences are present, and that flip-flop inversions in each pair of the IRs appear to generate four distinct $\mathrm{mt}$ genome structures.

\section{Results and discussion}

\section{Mitochondrial genome organization}

We obtained the complete $\mathrm{mt}$ genome sequences of B. microti (Munich strain) and B. rodhaini (Australian strain). The B. microti mt genome was a monomeric linear molecule of $11.1 \mathrm{~kb}$ (Figures 1-A and 2-A) and contained three protein-coding genes, $c o x 1, c o b$ and cox3, and seven SSU and 12 LSU rRNA gene fragments (Figure 1-A). Unexpectedly, the B. microti mt genome possessed two pairs of long inverted repeats, inverted repeats A (IR-A) and B (IR-B). The nucleotide sequences of IR-A (817 bp) and IR-B (1082 bp) were dissimilar to each other. A 3.4-kb region between two IR-As contained cox1, RNA18, RNA6, RNA14, RNA15, LSU4, LSU5, cob, LSU2 and LSU6, and a 2.9-kb region between two IR-Bs contained RNA2, cox3, RNA17, SSUE, SSUF, LSU1, LSU3, SSUA, LSUB, SSUD, SSUB, LSUA and RNA1. The predicted secondary structures for fragments comprising the B. microti SSU and LSU rRNA, except for the LSU1-LSU6 whose secondary structures have been predicted in Theileria parva and Babesia gibsoni [8,17], are shown in Additional file 1: Figure S1-A. RNA15 is a transcript of unknown function in Plasmodium falciparum and T. parva [9]. An intervening region (1080 bp) between IR-A and IR-B does not appear to contain any gene or gene fragment. Searches for other repeat sequences identified additional three short repeats with lengths of 23, 65 and $103 \mathrm{bp}$ in the $B$. microti $\mathrm{mt}$ genome (Figure 1-A and Additional file 2: Table S1).

Interestingly, the $B$. microti $\mathrm{mt}$ genome displayed four distinct genome structures, types I, II, III and IV (Figure 1-A). The four genome structures can be generated by two inversions: one is an inversion of a region containing the 3.4-kb region between the IR-As (here termed Region A) and the other an inversion of a region containing the 2.9-kb region between the IR-Bs (Region B). Southern blot hybridization with probe $\operatorname{cox} 1$ (Bm-1) against undigested $B$. microti genomic DNA produced a clear signal at $11.1 \mathrm{~kb}$ (lane 1 in Figure 2-A). Hybridization against DNA digested with DraI gave two bands at $10.0 \mathrm{~kb}$ and $7.4 \mathrm{~kb}$ (lane 2). Hybridization against Eco065I-digested DNA yielded two bands at $8.9 \mathrm{~kb}$ and $2.8 \mathrm{~kb}$ (lane 3). These results are consistent with the hypothesis that the four genome structures, types I, II, III and IV are generated by dual 'flip-flop' inversions of Region A and Region B. Thus, DraI digestion produced $10-\mathrm{kb}$ and 1.1-kb fragments (types I and III in Figure 1-A), and additionally produced 7.4-kb and 3.7-kb fragments (types II and IV, if Region B was inverted): and Eco065I digestion produced $8.9-\mathrm{kb}, 2.2-\mathrm{kb}$ (types I and II in Figure 1-A) and $8.3-\mathrm{kb}$ and $2.8-\mathrm{kb}$ fragments (types III and IV, if Region A was inverted). Hybridization with a cox3 probe (Bm-2) revealed a clear signal at $11.1 \mathrm{~kb}$ against undigested genomic DNA (lane 4), two bands at $10.0 \mathrm{~kb}$ and $3.7 \mathrm{~kb}$ against DNA digested with DraI (lane 5), and two bands at $8.9 \mathrm{~kb}$ and $8.3 \mathrm{~kb}$ against DNA with Eco065I (lane 6). Another B. microti probe from an intervening region $(\mathrm{Bm}-3)$ gave a band at $11.1 \mathrm{~kb}$ against undigested genomic DNA (lane 7), two bands at 10.0 and $7.4 \mathrm{~kb}$ against DraItreated DNA (lane 8), and two bands at $8.9 \mathrm{~kb}$ and $8.3 \mathrm{~kb}$ against Eco065I-digested DNA (lane 9). These results obtained with $\mathrm{Bm}-2$ and $\mathrm{Bm}-3$ are consistent with the idea of dual 'flip-flop' inversions. 
A

Type-I

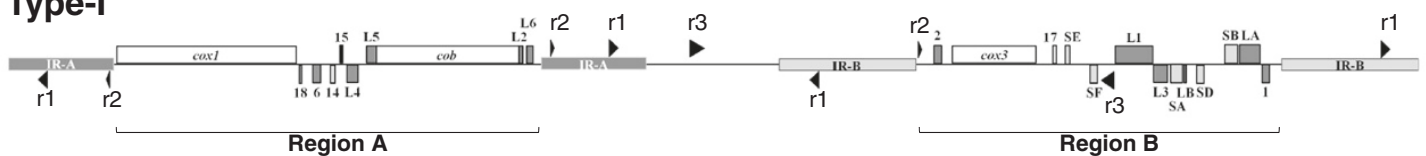

Type-II

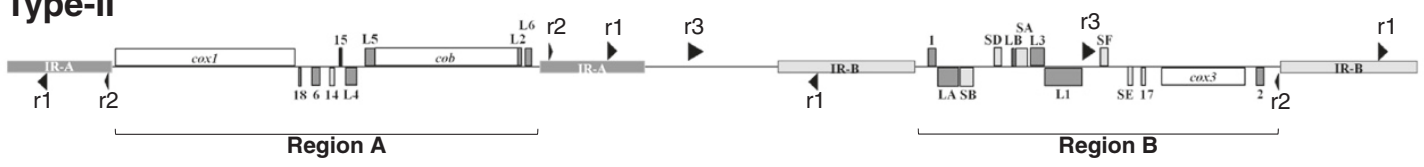

Type-III

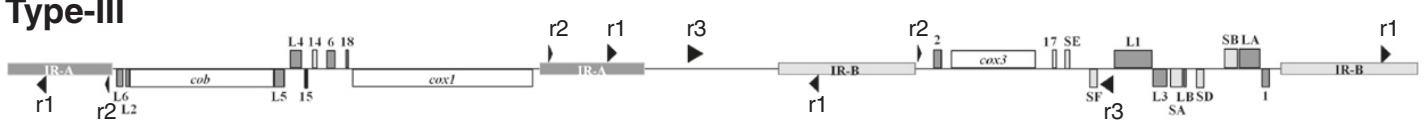

Region A

Region B

\section{Type-IV}

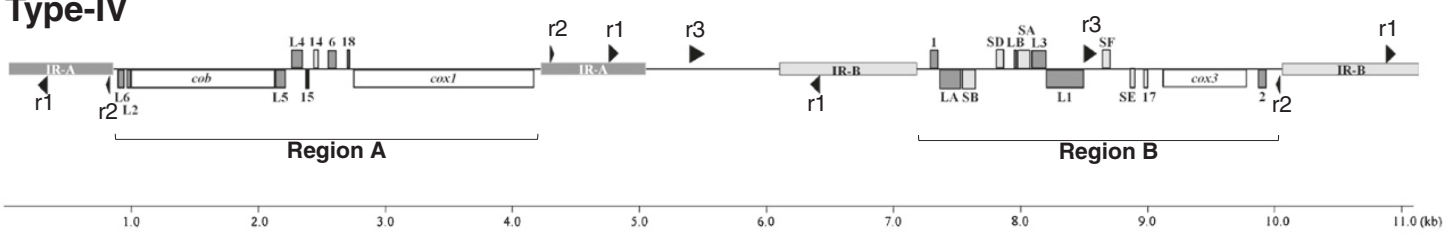

B

\section{Type-I}

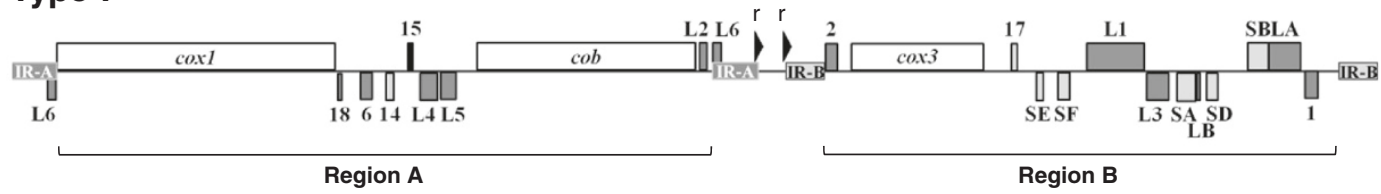

Type-II
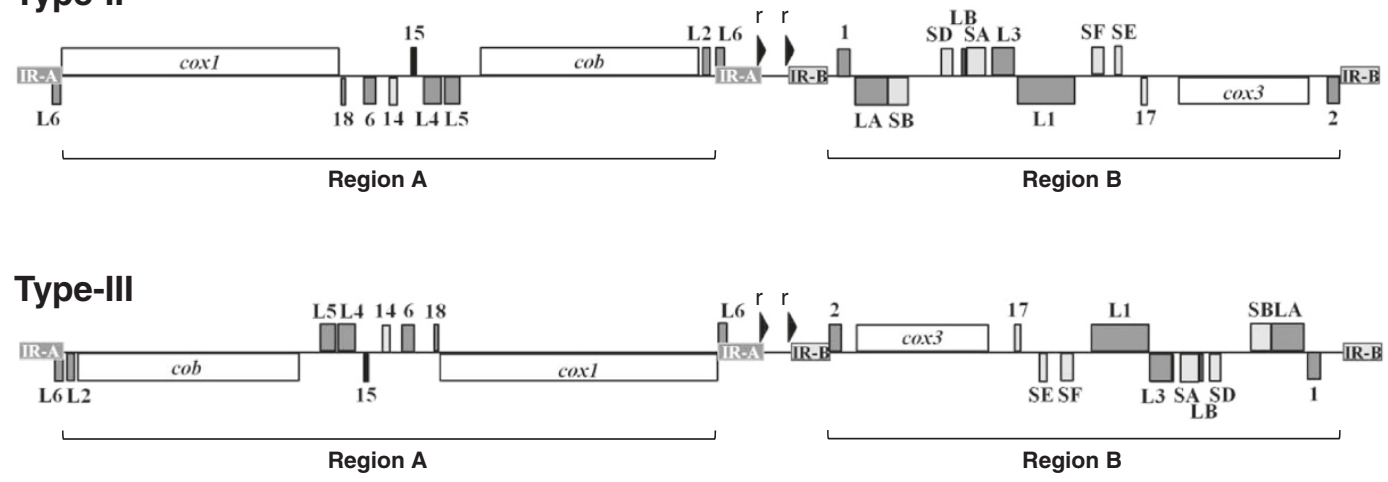

Type-IV

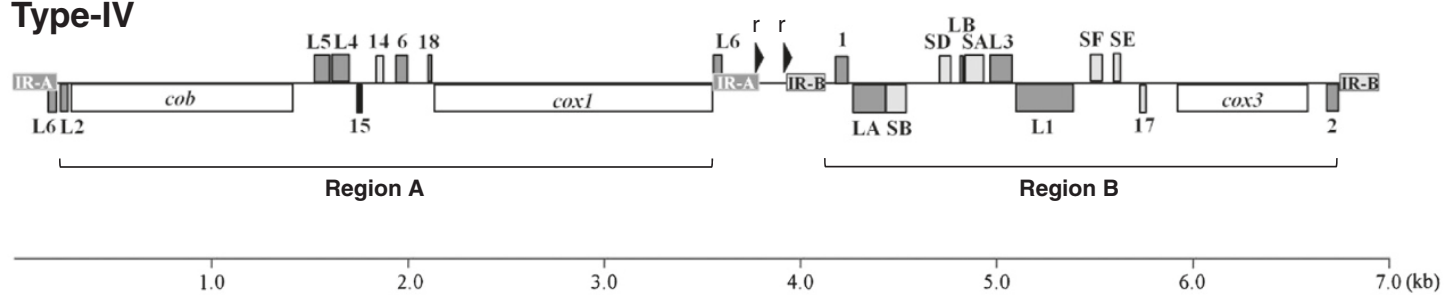

Figure 1 (See legend on next page.) 
(See figure on previous page.)

Figure 1 Four distinct mitochondrial (mt) genome structures in Babesia microti (A) and Babesia rodhaini (B). These mt genomes possess two pairs of inverted repeats, IR-A and IR-B. Genes shown above bold line are transcribed from left to right and those below from right to left. Light and dark gray blocks indicate fragments of small subunit (SSU) and large subunit (LSU) rRNA genes, respectively. Abbreviations: cox1, cytochrome c oxidase subunit 1 gene; cox3, cytochrome c oxidase subunit 3 gene; cob, cytochrome $b$ gene. Black arrowheads, r1, r2 and r3 in the B. microti $\mathrm{mt}$ genome and $\mathrm{r}$ in the B. rodhaini $\mathrm{mt}$ genome, indicate short direct or inverted repeat sequences (see Additional file 2 : Table S1 and Additional file 1: Figure S4).

The B. rodhaini mt genome $(6.9 \mathrm{~kb})$ also possessed two pairs of long inverted repeats, IR-A (220 bp) and IR$\mathrm{B}(184 \mathrm{bp})$ and four distinct genome structures (types I, II, III and IV) (Figure 1-B). IR-A contained LSU6. A 3.3-kb region between two IR-As (Region A) contained cox 1 , RNA18, RNA6, RNA14, RNA15, LSU4, LSU5, cob and LSU2. A 2.6-kb region between two IR-Bs (Region B) contained RNA2, cox3, RNA17, SSUE, SSUF, LSU1, LSU3, SSUA, LSUB, SSUD, SSUB, LSUA and RNA1. The transcriptional direction of SSUE and LSU5 of the $B$. rodhaini $\mathrm{mt}$ genome is different from that of the $B$. microti mt genome. The predicted secondary structures for fragments comprising the B. rodhaini SSU and LSU rRNA, except for the LSU1-LSU6, are shown in Additional file 1: Figure S1-B. RNA15 seems to be functionally important since its nucleotide sequence is highly conserved among B. microti, B. rodhaini, T. parva and $P$. falciparum (Additional file 1: Figure S2). An intervening region (152 bp) between IR-A and IR-B does not appear to contain any gene and gene fragment. In addition to the two IRs, a pair of short direct repeat was identified (Figure 1-B and Additional file 2: Table S1).

As in the B. microti mt genome, the four genome structures of $B$. rodhaini can also be generated by dual flip-flop inversions of Region A and Region B. Thus, hybridization with $\mathrm{Br}-1$ against undigested DNA produced a clear signal at $6.9 \mathrm{~kb}$ (lane 1 in Figure 2-B), as expected from the genome sequence. Hybridization against HindIII-digested DNA gave two bands at 6.6 and $3.5 \mathrm{~kb}$ (lane 2), the former being expected in types III and IV (Figure 1-B), and the latter expected in types I and II (Figure 1-B). Hybridization against XhoI-digested DNA yielded two bands at 6.0 and $4.8 \mathrm{~kb}$, (lane 3), the former being expected in types I and III, and the latter expected in types II and IV. Hybridization with $\mathrm{Br}-2$ yielded a band at $6.9 \mathrm{~kb}$ against undigested DNA (lane 4), two bands at 6.6 and $3.4 \mathrm{~kb}$ against HindIII-digested DNA (lane 5), and two bands at 6.0 and $2.0 \mathrm{~kb}$ against XhoI-digested DNA (lane 6). The intervening region probe (Br-3) gave a band at $6.9 \mathrm{~kb}$ against undigested DNA (lane 7), two bands at 6.6 and $3.4 \mathrm{~kb}$ against HindIIIdigested DNA (lane 8), and two bands at 6.0 and $4.8 \mathrm{~kb}$ against XhoI-digested DNA (lane 9). These signals were consistent with the four genome structures (Figure 2-B).

All monomeric linear $\mathrm{mt}$ genomes characterized to date have a single pair of TIR on both ends [5]. Thus, to our knowledge this is the first study to show two pairs of distinct IR sequences. In both B. microti and B. rodhaini, dual flip-flop inversions of Region A and Region B appear to generate four $\mathrm{mt}$ genome structures. We postulate that the dual flip-flop inversions are mediated through recombination in palindromes of IR-A and IR-B (Figure 3). Recombination between a pair of IR-As (and IR-Bs) produces an isomeric form characterized by a flip-flop of Region A (and Region B), thus generating four distinct $\mathrm{mt}$ genome structures.

Flip-flop inversion of nuclear or organelle genomes have been found in some organisms. In the bacterium Staphylococcus aureus, large-scale inversion of its chromosome switches on or off different phenotypes, including the expression of dozens of genes [18]. The mt activity of the Plasmodium genus, which is closely related to the Babesia and Theileria genera, has been reported to be different between mosquito stages and vertebrate stages [19]. Therefore, it is likely that flip-flop inversions of the $B$. microti and $B$. rodhaini mt genomes may switch on or off expression of $\mathrm{mt}$ genes and gene fragments in their lifecycles.

\section{Transcription}

RT-PCR using three separate primer sets targeting about 500-bp sequences of cox $1, \operatorname{cox} 3$ and $c o b$ of $B$. microti gave the expected transcript size using cDNA but not RNA (Additional file 1: Figure S3). Similarly, expected PCR sized fragments were obtained using primers specific to $B$. rodhaini for $\operatorname{cox} 1, \operatorname{cox} 3$ and $\operatorname{cob}$ (Additional file 1: Figure S3). Results confirm the transcription of the three protein-coding genes. We were unable to perform additional transcription analysis, such as a northern blotting, for 19 SSU and LSU gene fragments, due to extreme difficulties in obtaining an adequate amount of parasites from infected mice. Two reports of Kairo et al. [17] on the transcription of five LSU rRNA gene fragments (LSU1-LSU5) in T. parva and Hikosaka et al. [8] on the transcription of LSU6 in B. gibsoni, however, suggest that at least these six fragments are transcribed in B. microti and B. rodhaini.

\section{Estimations of the molar ratio of the four mt genome structures and $\mathrm{mt}$ genome copy number}

We estimated the molar ratio of the four genome structures of $B$. microti. The intensity ratios of the two signals 


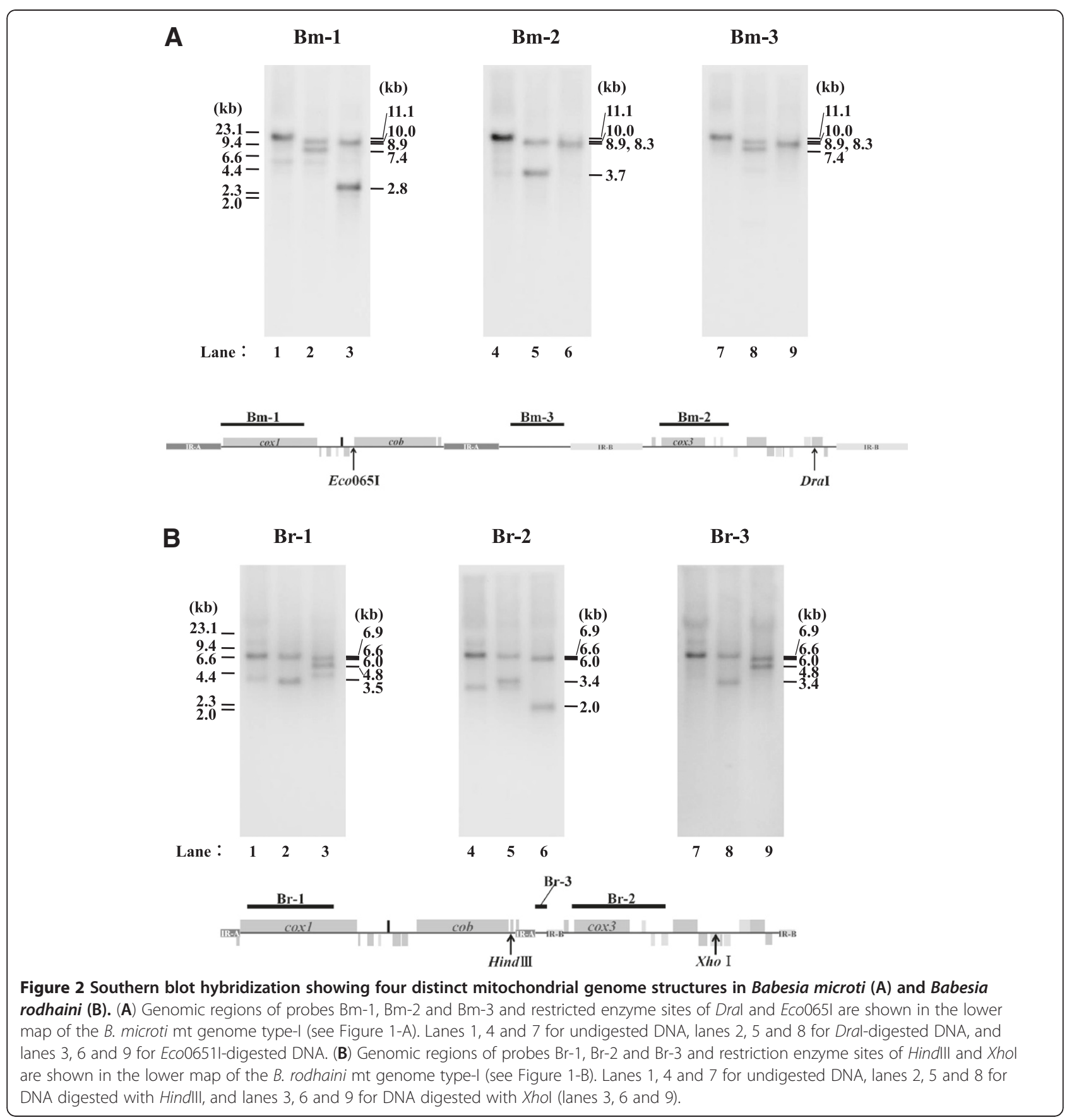

produced by hybridization of the Bm-1 probe with DraIdigested DNA (lane 2 in Figure 2-A) and Eco065Idigested DNA (lane 3 in Figure 2-A) were 0.7 and 0.8 (Additional file 2: Table S2), respectively. Likewise, hybridization of the $\mathrm{Bm}-2$ probe with DraI-digested DNA (lane 5 in Figure 2-A), and the Bm-3 probe with DraI-digested DNA (lane 8 in Figure 2-A) produced same signal intensities of 0.8 each. These suggest that a molar ratio of types-I, II, III and IV of the B. microti mt genome is approximately 1:1:1:1. We infer that the four distinct genome structures is generated from one parasite because the four genome structures was confirmed by Southern blot analysis for genomic DNA extracted from parasites derived from a single parasite cloned by limiting dilutions (data not shown). In addition, copy number analysis using Southern hybridization estimated about 20 copies of the mt genome per haploid nuclear genome. Taken together, these findings suggest that one parasite possesses four types of $\mathrm{mt}$ genome structure in B. microti. 


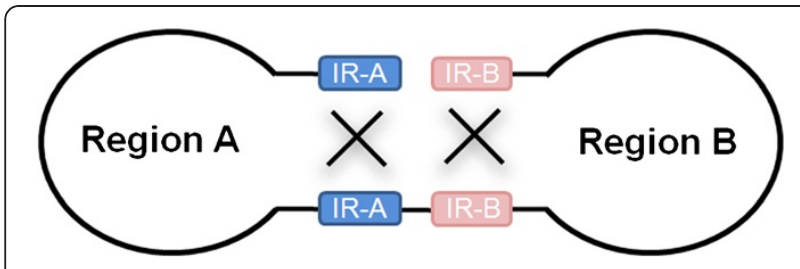

Figure $3 \mathrm{~A}$ postulated mechanism for inversions of Region $\mathrm{A}$ and Region B. Recombination between two IR-As (or two IR-Bs) produces an isomeric form characterized by a flip-flop of Region A (or Region B). Dual flip-flop inversions of IR-A and IR-B can generate four distinct genome structures.

In $B$. rodhaini, the molar ratio of the four mt genome structures (types I, II, III and IV) was also approximately $1: 1: 1: 1$. Thus, intensity ratio of the two signals produced in each case by hybridization of the $\mathrm{Br}-1$ probe with DNA digested by HindIII or XhoI (lanes 2 and 3 in Figure 2-B), and of the $\mathrm{Br}-2$ probe (lanes 5 and 6 in Figure 2-B), and of the $\mathrm{Br}-3$ probe (lanes 8 and 9 in Figure 2-B) ranged from 0.9 to 1.2 (Additional file 2: Table S2). The equivalent molar ratio was confirmed by Southern blot analysis using DNA of B. rodhaini cloned by limiting dilutions (data not shown). Copy number of the $B$. rodhiani mt genome was estimated to be two to three copies per haploid nuclear genome. This suggests that one parasite has one to three types of $\mathrm{mt}$ genome structures, and four distinct mt genome structures may be generated during cell proliferation.

\section{Phylogeny}

Both $B$. microti and B. rodhaini belong to an ancestral group of Babesia/Theileria, represented by the Archaeopiroplasmid group, according to phylogenetic analysis using 18S rRNA gene sequence [16]. The maximum likelihood (ML) tree inferred from concatenated COX1 and $\mathrm{COB}$ amino acid sequences (Figure 4) revealed a monophyletic relationship between Babesids, Theilerids and Archaeopiroplasmids with a bootstrap proportion (BP) value of $100 \%$, clearly separated from a clade of Plasmodium species. B. microti and B. rodhaini were grouped into a clade (BP value 100\%), which was located at the branch leading to the common ancestor of Babesids and Theilerids. These results indicate that the monomeric linear $\mathrm{mt}$ genomes found in the group of Babesids, Theilerids and Archaeopiroplasmids were generated specifically in this lineage.

\section{Conclusions}

We found a novel linear monomeric mt genome structure in the rodent piroplasms, Babesia microti and Babesia rodhaini, equipped with dual flip-flop inversion system, by which four distinct genome structures are readily generated. Such a unique linear $\mathrm{mt}$ genome structure has not been known in not only apicomplexan parasites but also in other organisms. The present findings would provide insight into further understanding of evolution of mt genome structure.

\section{Methods}

Animals

Four-week-old female ICR mice were purchased from Japan SLC (Shizuoka, Japan) and housed in microisolator cages within a modified pathogen-free barrier facility at the Animal Resource Center for Infectious Diseases, Research Institute for Microbial Diseases, Osaka University. All animals had free access to food and water ad libitum, and all of the experimental procedures followed our institutional guidelines.

\section{DNA sequencing}

B. microti (Munich strain) and B. rodhaini (Australian strain), were maintained by routine passage through mice. Infected blood was collected by cardiac puncture. Leukocytes were removed using Plasmodipur filters (EuroProxima, Arnhem, the Netherlands) [21]. Parasite genomic DNA was extracted using a QIAamp DNA Blood Mini Kit (Qiagen, Hilden, Germany) according to the manufacturer's instructions. The mt genomes of $B$. microti and B. rodhaini were directly sequenced using specific primers (Additional file 2: Table S3-A). Primers were designed by aligning reported $\mathrm{mt}$ genome sequences of Plasmodium falciparum (DDBJ/EMBL/ GenBank accession \# M76611), Plasmodium mexicanum (EF079653), Plasmodium yoelii (MALPY00209), Babesia bovis (AB499088), Theileria annulata (NW_001091933), Theileria equi (AB499091) and Theileria parva (AB499089). Mt DNA was amplified in a $20 \mu$ l reaction mixture containing $0.2 \mu \mathrm{M}$ each of forward and reverse primers, $400 \mu \mathrm{M}$ each of dNTP, 1 unit of LA-Taq (Takara Bio, Shiga, Japan), $2 \mu$ l of $10 \times$ PCR buffer, $2.5 \mathrm{mM}$ of $\mathrm{MgCl}_{2}$, and $1 \mu \mathrm{l}$ of genomic DNA. PCR conditions were as follows: initial denaturation at $94^{\circ} \mathrm{C}$ for $1 \mathrm{~min}$, and amplification for 40 cycles of $94^{\circ} \mathrm{C}$ for $30 \mathrm{~s}$, $55-68^{\circ} \mathrm{C}$ (depending on primers used) for $30 \mathrm{~s}$, and $72^{\circ} \mathrm{C}$ for 1-6 min, depending on amplification size $(1 \mathrm{~min}$ per $\mathrm{kb}$ ), followed by a final extension at $72^{\circ} \mathrm{C}$ for $10 \mathrm{~min}$.

Sequences of telomeric regions of the mt genomes of $B$. microti and B. rodhaini were determined by using the terminal deoxynucleotidyl transferase (TdT) tailing method [22] with minor modifications. Briefly, following initial denaturation of genomic DNA (150 ng) for $5 \mathrm{~min}$ at $95^{\circ} \mathrm{C}$, the 3 -ends was tailed with cytosine for $30 \mathrm{~min}$ at $37^{\circ} \mathrm{C}$ in a reaction mixture containing $200 \mu \mathrm{M} \mathrm{dCTP}$, $1 \mathrm{U}$ of TdT (Takara Bio), $20 \mathrm{mM}$ Tris- $\mathrm{HCl}(\mathrm{pH} 8.4$ ), $50 \mathrm{mM} \mathrm{KCl}$, and $1.5 \mathrm{mM} \mathrm{MgCl}_{2}$, and then heatinactivated at $65^{\circ} \mathrm{C}$ for $10 \mathrm{~min}$. The first PCR was done in a $50-\mu$ l reaction mixture containing $2 \mu \mathrm{l}$ of the tailed DNA fragments, 1.25 units of AmpliTaq DNA 


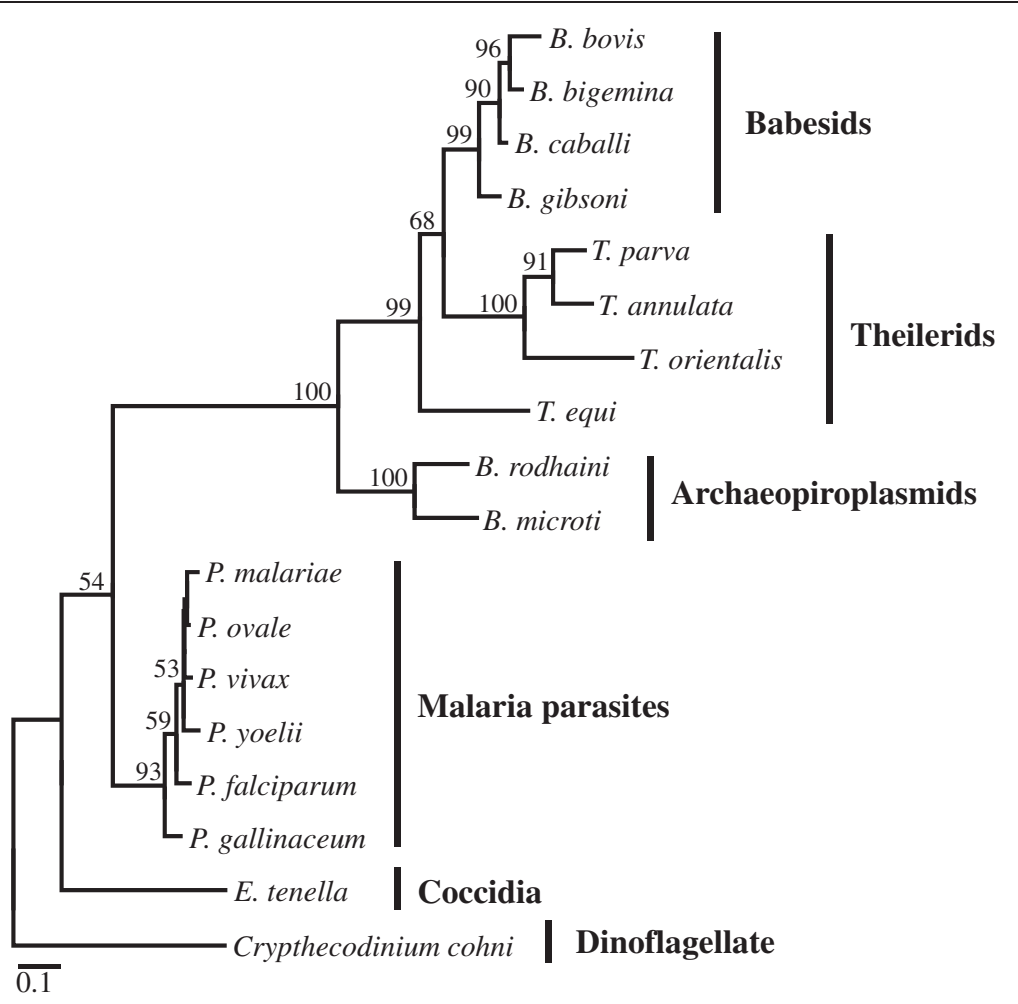

Figure 4 The ML phylogenetic tree of mitochondrial protein coding genes, cox 1 and cob, from 17 apicomplexans. Crypthecodinium cohnii was used as an outgroup. Concatenated amino acid sequences (696 sites) were used with 1,000 heuristic replicates under a Jones Taylor, and Thornton model [20] $(\alpha=0.69)$ for constructing this tree. Numbers shown along nodes represent bootstrap values.

Polymerase (Applied Biosystems, Life Technologies, Carlsbad, CA), $2.5 \mathrm{mM} \mathrm{MgCl} 2,200 \mu \mathrm{M}$ dNTPs, $0.4 \mu \mathrm{M}$ of a mt genome-specific primer (Additional file 2: Table S3-B) and a selective anchor primer (5'-CTACTACTAC TAGGCCACGCGTCGACTAGTACGGGGGGGGGGGGGG GG-3'). The PCR was performed by initial denaturation at $95^{\circ} \mathrm{C}$ for $2 \mathrm{~min}$, and 40 cycles of $94^{\circ} \mathrm{C}$ for $30 \mathrm{~s}, 62^{\circ} \mathrm{C}$ for $3 \mathrm{~min}$, followed by an extension step at $72^{\circ} \mathrm{C}$ for $10 \mathrm{~min}$. For each sample, $1 \mu \mathrm{l}$ of the first PCR products was used for the nested PCR amplification in a 50- $\mu$ l reaction mixture as mentioned above, containing a nested primer (Additional file 2: Table S3-B) and a universal amplification primer (5'-CTACTACTACTAGGCCACGCGTCGA CTAGTAC-3'). The second PCR was performed by initial denaturation at $95^{\circ} \mathrm{C}$ for $2 \mathrm{~min}$, and 25 cycles of $94^{\circ} \mathrm{C}$ for $30 \mathrm{~s}, 62^{\circ} \mathrm{C}$ for $2 \mathrm{~min}$, followed by an extension step at $72^{\circ} \mathrm{C}$ for $10 \mathrm{~min}$. PCR products were purified using QIAquick PCR purification kit (Qiagen), and sequenced directly from two independent PCR products, using the BigDye $^{\circledR}$ Terminator v3.1 Cycle Sequencing Kit (Applied Biosystems, Life Technologies) in an ABI 3130 Genetic Analyzer (Applied Biosystems, Life Technologies). Sequencing primers were designed to cover target regions in both directions. The sequences obtained in this study have been deposited in DDBJ/EMBL/GenBank with the following accession numbers, AB624353 - AB624356 (B. microti mt genome structures type-I to type-IV) and AB624357 - AB624360 (B. rodhaini mt genome structures type-I to type-IV).

\section{Gene annotation}

Nucleotide sequences of the mt genomes from $B$. microti and $B$. rodhaini and their deduced amino acid sequences were aligned with reported sequences from $P$. falciparum (M76611), B. bovis (AB499088), T. annulata (NW_001091933), T. equi (AB499091) and T. parva (AB499089) by Clustal W [23] with manual correction. Protein-coding regions were inferred using previously annotated sequences from $T$. parva and B. bovis.

To identify putative rRNA genes, mt DNA sequences or annotated rRNA gene fragments from $B$. bovis (EU075182) and T. parva (Z23263) were used as a query under suggested algorithm parameters [24] in NCBI BLAST 2.2 [25]. In silico analysis was also performed with Probalign beta version 1.2 [26] and SSEARCH 3.5 [27] using known rRNA gene fragments and suggested advanced search options [24,26]. Newly identified candidate rRNA genes were, likewise, used as input sequences. Information from sequence alignments using CLUSTAL W [23] and putative base-pairings between fragments 
proposed for $T$. parva mt rRNA fragments [9,17] were used to determine the termini of candidate rRNA genes.

\section{Search for repeat sequences}

Repeat sequences were searched using the REPFIND program (http://zlab.bu.edu/repfind/) [28] with cut-off of $>20$ nucleotides and a $P$-value $<0.0001$. Inverted repeat sequences were searched using a 'self against self' BLASTN search [29] with cut-off of $>20$ nucleotides. Additional searches for repeats and inverted repeats were performed using GENETYX soft ware (Version 8; SDC, Tokyo, Japan).

\section{Southern blot hybridization}

Genomic DNA of B. microti, either undigested or digested with DraI or Eco065I, and that of B. rodhaini, either undigested or digested with HindIII or XhoI, were electrophoresed on $0.8 \%(\mathrm{w} / \mathrm{v})$ agarose gels in TAE (40 mM Tris-acetate, $1 \mathrm{mM}$ EDTA) and then transferred to a positively charged nylon membrane (Amersham Hybond-N+, GE Healthcare, Little Chalfont, England). Specifically amplified PCR products from B. microti and $B$. rodhaini genomic DNA (Additional file 2: Table S3-C) were labeled with digoxigenin-dUTP using the DIG High Prime DNA Labeling and Detection Starter Kit II (Roche Diagnostics, Rotkreuz, Switzerland). The DIG-labeled DNA probes were incubated with the nylon membrane, and blots were washed twice with $2 \times$ SSC, $0.1 \%$ SDS and twice with $0.5 \times$ SSC, $0.1 \%$ SDS, at $65^{\circ} \mathrm{C}$ for $15 \mathrm{~min}$. Hybridization signals were detected using the Detection Starter Kit II. Chemiluminescence signals were quantitated using LAS-4000mini (GE Healthcare BioSciences AB, Uppsala, Sweden).

\section{RNA preparation and analysis}

Transcription of cox $1, \operatorname{cox} 3$ and $c o b$ in B. microti and B. rodhaini was analyzed by RT-PCR. Total RNA was extracted with RNeasy Mini Kit (Qiagen). Residual DNA in the RNA preparation was removed by DNase I treatment. cDNA synthesis and DNA amplification were carried out using specific primers (Additional file 2: Table S2-D) with PrimeScript ${ }^{\circledR}$ High Fidelity RT-PCR Kit (Takara Bio). RNA extracts that were not treated with reverse transcriptase gave no PCR products.

\section{Copy number estimation}

Copy numbers of mt genomes of B. microti and B. rodhaini were estimated using dot blot hybridization. Briefly, DNA fragments of the mt genome and of the B. microti and B. rodhaini beta-tubulin genes (nuclear genome) were amplified by PCR using specific primers (Additional file 2: Table S3-C), and DNA amount was measured. Serial dilutions of control PCR products of known DNA amounts were dot-blotted onto a nylon membrane, following heat denaturation $\left(99^{\circ} \mathrm{C}, 10 \mathrm{~min}\right)$. Genomic DNA were electrophoresed on agarose gels and then transferred to a nylon membrane. A PCR product specifically amplified from target regions of the $\mathrm{mt}$ genome and the beta-tubulin gene was labeled as described. Chemiluminescence signals were quantitated using LAS-4000mini.

\section{Phylogenetic analysis}

Concatenated amino acid sequences of COX1 and $\mathrm{COB}$ (696 sites) from 17 apicomplexan parasites (Additional file 2: Table S4) were used for phylogenetic analysis. A free living dinoflagellate, Crypthecodinium cohnii [30,31], was included as an outgroup. COX3 were not used for phylogenetic analysis, due to very high divergence in Babesia/Theileria species [8]. We constructed the ML phylogenetic tree by the PROML program in PHYLIP version 3.68 [32]. CODEML program in PAML version 4.2 [33] was used to estimate the $\Gamma$ shape parameter value $\alpha$. Bootstrap analysis was done by applying PROML to 100 re-sampled datasets produced by SEQBOOT program in PHYLIP. BP values were calculated for internal branches of the ML-tree using CONSENSE in PHYLIP.

\section{Additional files}

Additional file 1: This file contains Supplemental Figures S1-S4.

Additional file 2: This file contains Supplemental Tables S1-S4.

\section{Abbreviations}

mt genome: Mitochondrial genome; kb: Kilobase; IR: Inverted repeats; TIR: Terminal inverted repeat; cox1: Cytochrome c oxidase subunit I gene; cox3: Cytochrome c oxidase subunit III gene; cob: Cytochrome b gene; LSU: Large subunit; rRNA: Ribosomal RNA; SSU: Small subunit; bp: Base pairs; ML: Maximum likelihood; BP: Bootstrap proportion; min: Minute; TdT: Terminal deoxynucleotidyl transferase; dCTP: Deoxycytidine 5/triphosphate.

\section{Competing interests}

The authors have declared that no competing interests exist.

\section{Authors' contributions}

$\mathrm{KH}$ carried out the molecular experiments. KT coordinated all experiments. $\mathrm{KH}, \mathrm{KK}$ and $\mathrm{KT}$ contributed to the research design. $\mathrm{KH}$ and $\mathrm{KT}$ drafted the manuscript. NT and II provided parasite samples. YW analyzed data. HK and TH provided critical comments about this study. All authors read and approved the final manuscript.

\section{Acknowledgements}

This work was supported by Grant-in-Aids for Scientific Research from Japan Society for Promotion of Sciences (18GS03140013 and 20390120) and Research Fellowships of the Japan Society for the Promotion of Science for Young Scientists. We thank E.O. Balogun for language corrections.

\section{Author details}

${ }^{1}$ Laboratory of Malariology, Research Institute for Microbial Diseases, Osaka University, 3-1 Yamadaoka, Suita, Osaka 565-0871, Japan. ²Department of Biomedical Chemistry, Graduate School of Medicine, The University of Tokyo, 7-3-1 Hongo, Bunkyo-ku, Tokyo 113-0033, Japan. ${ }^{3}$ Laboratory of Parasitic Diseases, National Institute of Animal Health, National Agriculture and Food 
Research Organization, Tsukuba, Ibaraki, Japan. ${ }^{4}$ Department of Molecular Biology, Research Institute for Microbial Diseases, Osaka University, Suita, Osaka, Japan. ${ }^{5}$ Department of Molecular Protozoology, Research Institute for Microbial Diseases, Osaka University, Suita, Osaka, Japan. ${ }^{6}$ National Research Center for Protozoan Diseases, Obihiro University of Agriculture and Veterinary Medicine, Obihiro, Hokkaido, Japan.

Received: 24 April 2012 Accepted: 29 October 2012

Published: 14 November 2012

\section{References}

1. Gray MW, Lang BF, Burger G: Mitochondria of protists. Annu Rev Genet 2004, 38:477-524.

2. Boore JL: Animal mitochondrial genomes. Nucleic Acids Res 1999, 27(8):1767-1780

3. Shao R, Kirkness EF, Barker SC: The single mitochondrial chromosome typical of animals has evolved into 18 minichromosomes in the human body louse, Pediculus humanus. Genome Res 2009, 19(5):904-912.

4. Palmer JD, Soltis D, Soltis P: Large size and complex structure of mitochondrial DNA in two nonflowering land plants. Curr Ggenet 1992, 21(2):125-129

5. Nosek J, Tomaska L: Mitochondrial genome diversity: evolution of the molecular architecture and replication strategy. Curr Genet 2003, 44(2):73-84.

6. Fan J, Lee RW: Mitochondrial genome of the colorless green alga Polytomella parva: two linear DNA molecules with homologous inverted repeat Termini. Mol Biol Evol 2002, 19(7):999-1007.

7. Burger $G$, Forget $L$, Zhu Y, Gray MW, Lang BF: Unique mitochondrial genome architecture in unicellular relatives of animals. Proc Natl Acad Sci USA 2003, 100(3):892-897.

8. Hikosaka K, Watanabe Y, Tsuji N, Kita K, Kishine H, Arisue N, Palacpac NM, Kawazu S, Sawai H, Horii T, Igarashi I, Kita K, Tanabe K: Divergence of the mitochondrial genome structure in the apicomplexan parasites, Babesia and Theileria. Mol Biol Evol 2010, 27(5):1107-1116.

9. Feagin JE, Harrel MI, Lee JC, Coe KJ, Sands BH, Cannone JJ, Tami G, Schnare MN, Gutell RR: The fragmented mitochondrial ribosomal RNAs of Plasmodium falciparum. PLoS One 2012, 7(6):e38320

10. Lau AO: An overview of the Babesia, Plasmodium and Theileria genomes: a comparative perspective. Mol Biochem Parasitol 2009, 164(1):1-8.

11. Feagin JE: Mitochondrial genome diversity in parasites. Int J Parasitol 2000, 30(4):371-390.

12. Hikosaka K, Watanabe Yl, Kobayashi F, Waki S, Kita K, Tanabe K: Highly conserved gene arrangement of the mitochondrial genomes of 23 Plasmodium species. Parasitol Int 2011, 60(2):175-180.

13. Feagin $J E$, Mericle BL, Werner $E$, Morris M: Identification of additional rRNA fragments encoded by the Plasmodium falciparum $6 \mathrm{~kb}$ element. Nucleic Acids Res 1997, 25(2):438-446.

14. Hikosaka K, Nakai Y, Watanabe YI, Tachibana SI, Arisue N, Palacpac NM, Toyama T, Honma H, Horii T, Kita K, Tanabe K: Concatenated mitochondrial DNA of the coccidian parasite Eimeria tenella. Mitochondrion 2011, 11 (2):273-278.

15. Lin RQ, Qiu LL, Liu GH, Wu XY, Weng YB, Xie WQ, Hou J, Pan H, Yuan ZG, Zou FC, Hu M, Zhu XQ: Characterization of the complete mitochondrial genomes of five Eimeria species from domestic chickens. Gene 2011, 480(1-2):28-33.

16. Criado-Fornelio A, Martinez-Marcos A, Buling-Sarana A, Barba-Carretero JC: Molecular studies on Babesia, Theileria and Hepatozoon in southern Europe. Part II. Phylogenetic analysis and evolutionary history. Vet Parasitol 2003, 114(3):173-194.

17. Kairo A, Fairlamb AH, Gobright E, Nene V: A 7.1 kb linear DNA molecule of Theileria parva has scrambled rDNA sequences and open reading frames for mitochondrially encoded proteins. EMBO J 1994, 13(4):898-905.

18. Cui L, Neoh H, Iwamoto A, Hiramatsu K: Coordinated phenotype switching with large-scale chromosome flip-flop inversion observed in bacteria. Proc Natl Acad Sci USA 2012, 109(25):E1647-E1656.

19. Hino A, Hirai M, Tanaka TQ, Watanabe Y, Matsuoka H, Kita K: Critical roles of the mitochondrial complex II in oocyst formation of rodent malaria parasite Plasmodium berghei. J Biochem 2012, 152(3):259-268.

20. Jones DT, Taylor WR, Thornton JM: The rapid generation of mutation data matrices from protein sequences. Comput Appl Biosci 1992, 8(3):275-282.
21. Janse CJ, Camargo A, Del Portillo HA, Herrera S, Kumlien S, Mons B, Thomas A, Waters AP: Removal of leucocytes from Plasmodium vivax-infected blood. Ann Trop Med Parasitol 1994, 88(2):213-216.

22. Bah A, Bachand F, Clair E, Autexier C, Wellinger RJ: Humanized telomeres and an attempt to express a functional human telomerase in yeast. Nucleic Acids Res 2004, 32(6):1917-1927.

23. Thompson JD, Higgins DG, Gibson TJ: CLUSTAL W: improving the sensitivity of progressive multiple sequence alignment through sequence weighting, position-specific gap penalties and weight matrix choice. Nucleic Acids Res 1994, 22(22):4673-4680

24. Freyhult EK, Bollback JP, Gardner PP: Exploring genomic dark matter: a critical assessment of the performance of homology search methods on noncoding RNA. Genome Res 2007, 17(1):117-125.

25. Altschul SF, Gish W, Miller W, Myers EW, Lipman DJ: Basic local alignment search tool. J Mol Biol 1990, 215(3):403-410.

26. Roshan U, Chikkagoudar S, Livesay DR: Searching for evolutionary distant RNA homologs within genomic sequences using partition function posterior probabilities. BMC Bioinforma 2008, 9:61.

27. Pearson WR: Searching protein sequence libraries: comparison of the sensitivity and selectivity of the Smith-Waterman and FASTA algorithms. Genomics 1991, 11(3):635.

28. Betley JN, Frith MC, Graber JH, Choo S, Deshler JO: A ubiquitous and conserved signal for RNA localization in chordates. Curr Biol 2002, 12(20):1756.

29. Altschul SF, Madden TL, Schaffer AA, Zhang J, Zhang Z, Miller W, Lipman DJ: Gapped BLAST and PSI-BLAST: a new generation of protein database search programs. Nucleic Acids Res 1997, 25(17):3389-3402.

30. Norman JE, Gray MW: A complex organization of the gene encoding cytochrome oxidase subunit 1 in the mitochondrial genome of the dinoflagellate, Crypthecodinium cohnii: homologous recombination generates two different cox1 open reading frames. J Mol Evol 2001, 53(4-5):351-363.

31. Zhang $\mathrm{H}$, Lin S: Mitochondrial cytochrome $b$ mRNA editing in dinoflagellates: possible ecological and evolutionary associations?. J Eukaryot Microbiol 2005, 52(6):538-545.

32. Felsenstein J, Churchill GA: A hidden markov model approach to variation among sites in rate of evolution. Mol Biol Evol 1996, 13(1):93-104.

33. Yang Z: PAML: a program package for phylogenetic analysis by maximum likelihood. Comput Appl Biosci 1997, 13(5):555-556.

\section{doi:10.1186/1471-2164-13-622}

Cite this article as: Hikosaka et al:: Novel type of linear mitochondrial genomes with dual flip-flop inversion system in apicomplexan parasites, Babesia microti and Babesia rodhaini. BMC Genomics 2012 13:622.

\section{Submit your next manuscript to BioMed Central and take full advantage of:}

- Convenient online submission

- Thorough peer review

- No space constraints or color figure charges

- Immediate publication on acceptance

- Inclusion in PubMed, CAS, Scopus and Google Scholar

- Research which is freely available for redistribution 